\title{
Recovering of oxygenation, physical function and disability in patients with Covid-19
}

\author{
Laura Bertacchini ${ }^{1}$, Mara Paneroni ${ }^{1}$, Laura Comini ${ }^{2}$, Simonetta Scalvini ${ }^{3}$, Michele Vitacca ${ }^{1}$ \\ ${ }^{1}$ Respiratory Rehabilitation, ICS Maugeri IRCCS, Institute of Lumezzane (BS); ${ }^{2}$ Scientific Direction, ICS Maugeri \\ IRCCS, Institute of Lumezzane (BS); ${ }^{3}$ Care Continuity Unit and Telemedicine Service, ICS Maugeri IRCCS, Institute of \\ Lumezzane (BS), Italy
}

\begin{abstract}
The present case report describes middle-time course of respiratory and physical variables in eight Covid-19 patients who were transferred from ICU of Covid Hub in our subacute Covid-19 unit. Secondly they were admitted in a pulmonary rehabilitation unit and, at discharge, a tele-rehabilitation program was provided as a continuum of care at home.

Time course of oxygenation, physical function and disability were recorded.
\end{abstract}

\footnotetext{
Correspondence: Michele Vitacca, Istituti Clinici Scientifici Maugeri, IRCCS, Via G. Mazzini 129, 25065 Lumezzane (BS), Italy.

E-mail: michele.vitacca@icsmaugeri.it

Authors' contribution: All the authors have read and approved the final version of the manuscript and agreed to be accountable for all aspects of the work.

Acknowledgements: The authors would like to thank the PT team of the Cardio-Pulmonary Gym for collection of the data.

Funding: This work was supported by the Ricerca Corrente funding scheme of the Ministry of Health, Italy.
}

Conflict of interest: The authors declare no potential conflict of interest.

Informed consent: The manuscript does not contain any individual person's data in any form.

Availability of data and materials: All data generated or analyzed during this study are included in this published article.

Keywords: disability; coronavirus; rehabilitation; outcome; physical function.

Received for publication: 23 February 2021

Accepted for publication: 29 March 2021.

CCopyright: the Author(s), 2021

Licensee PAGEPress, Italy

Monaldi Archives for Chest Disease 2021; 91:1817

doi: 10.4081/monaldi.2021.1817

This article is distributed under the terms of the Creative Commons Attribution Noncommercial License (by-nc 4.0) which permits any noncommercial use, distribution, and reproduction in any medium, provided the original author(s) and source are credited.
As expected, the acute event produced in these patients a dramatic worsening in oxygenation and physical activities, with a substantial improvement in oxygenation and mild disability after the sub-acute stay.

After rehabilitation program, the patients showed additional improvement in particular in physical function. Anyway, this recover was not complete for all patients.

The majority of Covid-19 survivors experienced ARF recovered oxygenation, physical function and disability within a median time of 137 days. A minority needs further follow up and rehabilitation maintenance due to incomplete recovery.

\section{Introduction}

Covid-19 disease may cause pneumonia, acute respiratory failure (ARF) [1-3], and prolonged functional impairment [4-6] requiring rehabilitation [7]. The present case report describes middle-time course of respiratory and physical variables in severe hospitalized Covid-19 patients who experienced ICU and were proposed for rehabilitations programs (CE 2440CE (04LU)].).

\section{Case Report}

Eight patients without any previous comorbidities with Covid-19-induced acute respiratory failure (ARF), severe acute respiratory distress syndrome (ARDS) $\left(\mathrm{SpO}_{2} / \mathrm{FiO}_{2}=\right.$ $175.6 \pm 78.9)$ requiring invasive mechanical ventilation $(13 \pm 8$ days) ( 2 patients presented pulmonary embolism, 5 were submitted to pronation, all used azithromycin, chloroquine, steroids, anticoagulants, none were tracheostomized) were transferred from Covid-19 Hub where they had been managed in intensive care unit (ICU) to our Institute ICS Maugeri (Lumezzane, Italy) between April and June 2020 to prolong sub-acute and postacute recovery. They were immediately admitted in our subacute Covid-19 unit using pharmacological (steroids, anticoagulants, oxygen with a maximal oxygen $\mathrm{FiO}_{2}(\%)$ of $\left.0.40 \pm 0.12\right)$ and nonpharmacological care [8,9] consisting of individual session of early mobilization, active exercises and free walking, peripheral limb muscle activities, shoulder, and full arm circling, lung expansion treatments trough pronation therapy or positive expiratory pressure. Type, intensity, timing and modality of intervention were tailored to the individual patient according to age, clinical severity, length of immobilization with a daily session of 20 minutes. Patients were admitted in a pulmonary rehabilitation 
unit to be managed according to national and international rehabilitative procedures [9]: the program started from a minimum of one, 20 minute daily session up to two-three, 30 minute daily sessions in sessions with a physiotherapist/patient ratio 1:4-5. The program might include callisthenic, strengthening, balance exercise, paced walking. All exercises could be performed without devices or using gymnastic tools such as balls, canes, balance boards or light weights bands. Patients with higher physical autonomy were also trained on cycle-ergometer at low intensity exercises $(<3.0$ METs). The initial training workload was chosen starting from 0 progressively increasing until patients scored their dyspnea and/or leg fatigue as 4 or 5 on a modified 10-point Borg Scale. Thereafter the progression of intensity was according to Maltais et al. [10]: the workload was increased by 5 watts when patients scored less or equal to 3 , was unchanged when the Borg score was 4 or 5 and was reduced by 5 watts for scores of $>5$. Also, chest physiotherapy such as bronchial hygiene techniques by using disposable devices with self-management in order to avoid the risk of environmental contamination, and lung expansion procedures were performed when required. According to patient's individual conditions the program might include also nutritional and psychological assessment. At discharge from hospital, a tele-rehabilitation program was provided as a continuum of care at home: patients received a pulse oximeter, a brochure illustrating exercises, a diary to record daily activities, and instructions for home exercises. The one-month program consisted of one daily hour of aerobic reconditioning and muscle strengthening and healthy lifestyle education. Twice a week, a physiotherapist (PT) contacted the patient - by video-call via a dedicated platform - to monitor progress. Exercise intensity was based on baseline individual level of disability with low-intensity aerobics (walking, free-body exercise, sit-to-stand) and balance exercises. When patients presented an improvement in disability walking session with pedometer, aerobics with cycle ergometer or leg/arm crank, and strengthening exercises with a lightweight band were included.

Time course (Figure 1) $[\mathrm{T} 0=$ pre-Covid ARF according to data obtained anamnestically; T1 $=$ on the first 48 hours of ICU admission in acute hospital (range 2-10 days); T2 = at ICU discharge and post-acute admission at sub-acute ICS Maugeri Hospital (hospital setting) (range 33-71 days); T3 = beginning of rehabilitation (range 43-88 days); T4 = end of rehabilitation (range 64-144 days); T5 = end of tele-rehabilitation (range 94-174 days)] of oxygenation $\left(\mathrm{SpO}_{2} / \mathrm{FiO}_{2}\right)$, physical function [short physical performance battery test (SPPB) ][11] and disability (Barthel index)[12] were recorded. SPPB is a functional evaluation test comprehending 3 tests on balance, walking speed and the ability to rise from a chair 5 times, while Barthel index is an activities of daily living (ADL) scale.

Values along time course were calculated as variation in $\%$ to the pre-illness phase.

Table 1 shows individual parameters as mean $\pm \mathrm{SD}$ for all variables and along times.

Patients were $50 \%$ male, aged $67.5 \pm 10.7$ years, with a body mass index (BMI) of $26.9 \pm 3.7 \mathrm{Kg} / \mathrm{m}^{2}$ and no previous disability and diseases. In the initial phase (T1) (Figure 2a) oxygenation decreased on average by $61.3 \%$ and in a half of the patients it felt down more than $70 \%$ compared to the pre-Covid phase. During acute hospitalization (T1-T2), patients recovered on average by $26.4 \%$, reaching oxygenation values closer to the pre-illness phase at the end of subacute hospitalization. Compliance to the in-hospital rehabilitation sessions as for telerehabilitation was high (96\%).

At T4, the recovery was complete only in 3 patients (about $38 \%$ of the subjects). However, at T5, only one patient still had a significant oxygenation deficit ( $-26.7 \%$ compared to baseline). Observing the trend of physical function measured with SPPB test (Figure 2b), at T1, we found that 7 out of 8 patients had SPPB score equal to zero and 5 out of 8 had the same value at T2. At T3, patients had SPPB score equal to $37.7 \%$ of pre-Covid-19 values (SPPB score: $4.3 \pm 4.9$ ) and there were still 3 patients with a total score equal to 0 . At T4, patients, still presented severe deficits in physical function (SPPB score: $7.3 \pm 3.6,-35.9 \%$ from baseline= pre-Covid-19) and only 2 patients fully recovered. At T5 only 3 patients had no deficit, with an average value of SPPB (SPPB score: $10.0 \pm 2.1$ ) that remained at $88.5 \%$ of the pre-illness value. SPPB tests improved more were those relating to balance and walking speed. Regarding the time course of disability measured by Barthel Index (Figure 2c), a sharp drop of $85.1 \%$ was immediately noted, with a half of the patients having a score equal to 0 . At $\mathrm{T} 2$, there was a slight improvement with average values reaching $45.3 \%$ of the pre-Covid-19 values. At $\mathrm{T} 3$, a patient already reached values comparable to baseline, still having an average deficit of $30.8 \%$. At T4, 3 patients $(37.5 \%$ of the subjects) still had deficits, which were maintained for 2 of them during the tele-rehabilitation phase (T4-T5). At T5, therefore, still one patient had a significant disability $(-35 \%$ compared to the pre-illness phase).

Sub-items of Barthel Index referring of washing, toilet use and transfers improved more than others.

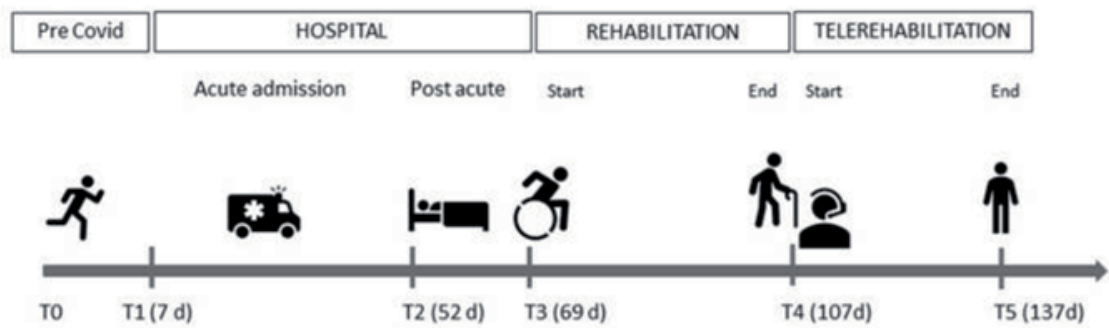

Figure 1. Times and settings of the patient's journey in which data were collected. T0, Pre-Covid ARF when patients presented good health; these data were obtained anamnestically; T1, on the first 48 hours of ICU admission in acute hospital; T2, at intensive care unit (ICU) discharge and post-acute admission at sub-acute ICS Maugeri Hospital (hospital setting); T3, beginning of rehabilitation at discharge from the sub-acute unit patients are admitted into the rehabilitation unit of the same institution (rehabilitation setting); $\mathrm{T} 4$, end of rehabilitation at discharge from the rehabilitation unit and introduced to a dedicated tele-rehabilitation program organized from the same Institution for caring patients at home; T5, end of telerehabilitation (rehabilitation setting) at the end of the tele-rehabilitation program. 


\section{Discussion}

Italian Covid-19 patients who developed ARF with or without hospitalization have been estimated to be about $43 \%$ of the total positive cases (personal unpublished data). Patients with Covid-19 after severe ARF, when admitted to our sub-acute unit presented a very low level of physical function (1.3 \pm 1.8 SPPB score) that was recovered within 4-5 months from the infection. Our data were similar to those reported in a previous study [13] in non Covid-19 ARF-survivor patients with SPPB scores $\leq 3.0$, in which physical function either did not recover within 6 months, or improved initially but survivors remained functionally disabled by 6 month [13].

As expected, the acute event with hospitalisation in ICU produced in these patients a dramatic worsening in oxygenation and physical activities during ADL with a substantial improvement in oxygenation and mild disability after the sub-acute stay.
After rehabilitation program, the patients showed additional improvement in particular in physical function and ability. Anyway, this recover was not complete for all patients (Figure 1). At the end of this progression program patients gained more in all aspects, but particularly they ameliorated their physical function.

Recently papers have been published warning the community of people after Covid-19 infection remaining symptomatic and with poor function [4-7]. The present study provides a middle-time course on oxygenation, physical function and disability in patients with Covid-19 from the most acute condition to home care; it offers some information on which rehabilitation programs has been proposed, on the amount of patients' function and disability improvement throw different settings and, finally, on how long was the recovery time.

Low sample size, difficulty to replicate our study, characteristics of the study design, patients excluded because they were lacking of data, lack of lung or respiratory muscle function tests and a

Table 1. Individual parameters as mean \pm SD for all variables and along times.

\begin{tabular}{|c|c|c|c|c|c|c|c|c|c|}
\hline & Patient 1 & Patient 2 & Patient 3 & Patient 4 & Patient 5 & Patient 6 & Patient 7 & Patient 8 & Overall (mean \pm SD) \\
\hline Age, y & 49 & 63 & 66 & 68 & 75 & 85 & 73 & 61 & $67.5 \pm 10.7$ \\
\hline BMI & 28.9 & 28.2 & 27.7 & 21.1 & 25.2 & 27.4 & 23.4 & 33.2 & $26.9 \pm 3.7$ \\
\hline $\mathrm{SpO}_{2} / \mathrm{FiO}_{2}, \mathrm{TO}$ & 457.1 & 452.4 & 447.6 & 461.9 & 452.4 & 447.6 & 457.1 & 461.9 & $454.8 \pm 5.7$ \\
\hline $\mathrm{SpO}_{2} / \mathrm{FiO}_{2}, \mathrm{Tl}$ & 102.5 & 111.3 & 118.6 & 103.3 & 316.7 & 204.4 & 213.3 & 235.0 & $175.6 \pm 78.9$ \\
\hline $\mathrm{SpO}_{2} / \mathrm{FiO}_{2}, \mathrm{~T} 2$ & 234.1 & 309.7 & 156.7 & 271.4 & 466.7 & 268.6 & 346.4 & 316.1 & $296.2 \pm 90.1$ \\
\hline $\mathrm{SpO}_{2} / \mathrm{FiO}_{2}, \mathrm{~T} 3$ & 395.8 & 357.7 & 303.2 & 282.9 & 466.7 & 339.3 & 342.9 & 316.1 & $350.6 \pm 58.3$ \\
\hline $\mathrm{SpO}_{2} / \mathrm{FiO}_{2}, \mathrm{~T} 4$ & 457.1 & 365.4 & 309.7 & 282.9 & 466.7 & 447.6 & 346.4 & 309.7 & $373.2 \pm 74.0$ \\
\hline $\mathrm{SpO}_{2} / \mathrm{FiO}_{2}, \mathrm{~T} 5$ & 452.4 & 461.9 & 447.6 & 452.4 & 461.9 & 466.7 & 336.7 & 461.9 & $445.6 \pm 43.7$ \\
\hline SPPB score, T0 & 12 & 12 & 12 & 12 & 7 & 12 & 12 & 12 & $11.4 \pm 1.8$ \\
\hline SPPB score, T1 & 0 & 0 & 0 & 2 & 0 & 0 & 0 & 0 & $0.3 \pm 0.7$ \\
\hline SPPB score, T2 & 0 & 0 & 0 & 4 & 2 & 4 & 0 & 0 & $1.3 \pm 1.8$ \\
\hline SPPB score, T3 & 10 & 0 & 1 & 11 & 3 & 9 & 0 & 0 & $4.3 \pm 4.9$ \\
\hline SPPB score, T4 & 12 & 3 & 7 & 12 & 5 & 10 & 5 & 4 & $7.3 \pm 3.6$ \\
\hline SPPB score, T5 & 12 & 9 & 11 & 12 & 7 & 11 & 7 & 11 & $10.0 \pm 2.1$ \\
\hline Barthel score, T0 & 100 & 100 & 100 & 100 & 90 & 100 & 100 & 100 & $98.8 \pm 3.5$ \\
\hline Barthel score, T1 & 0 & 0 & 0 & 0 & 40 & 20 & 25 & 30 & $14.4 \pm 16.4$ \\
\hline Barthel score, T2 & 40 & 20 & 10 & 90 & 65 & 65 & 20 & 45 & $44.4 \pm 27.6$ \\
\hline Barthel score, T3 & 85 & 40 & 55 & 100 & 80 & 95 & 35 & 55 & $68.1 \pm 25.1$ \\
\hline Barthel score, T4 & 95 & 65 & 100 & 100 & 90 & 100 & 50 & 100 & $87.5 \pm 19.3$ \\
\hline Barthel score, T5 & 100 & 100 & 100 & 100 & 90 & 100 & 65 & 100 & $94.4 \pm 12.4$ \\
\hline
\end{tabular}

$\mathrm{SpO}_{2} / \mathrm{FiO}_{2}$, relationship between the percentage of oxygen saturation and inspiratory fraction of oxygen; $\mathrm{SPPB}$, short physical performance battery.
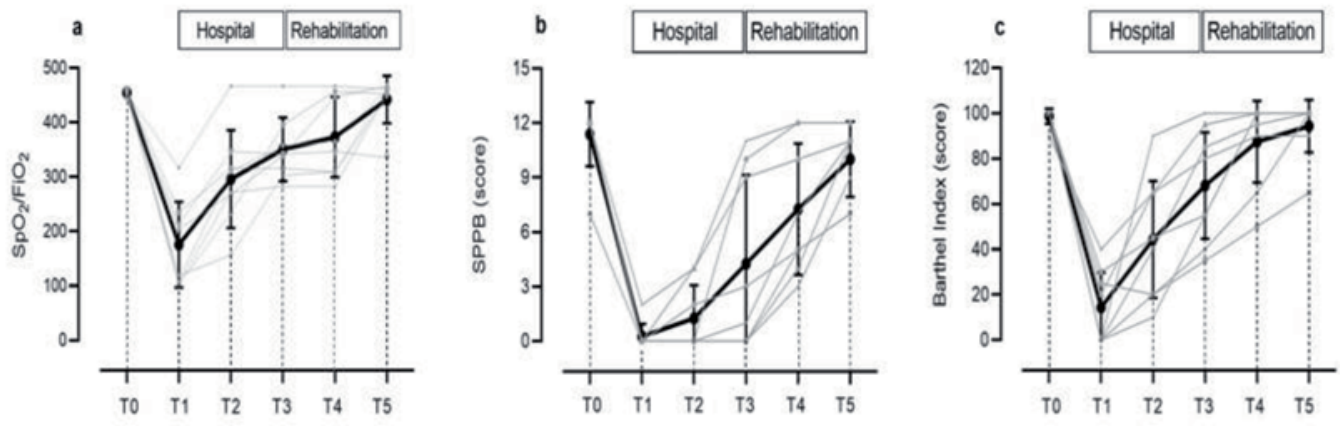

Figure 2. Trend of oxygenation (a), physical function (b) and disability (c) during the journey of the different patients (grey lines). For settings see the legend of Figure 1 . The mean curve $( \pm S D)$ is represented by the black bold line. $\mathrm{SpO}_{2} / \mathrm{FiO}_{2}$, relationship between the percentage of oxygen saturation and inspiratory fraction of oxygen; SPPB, short physical performance battery. 
real-time Covid-free control group as Covid patients without rehabilitation opportunities are limitations.

In conclusion, the majority of Covid-19 survivors experienced ARF due to pneumonia recovered oxygenation, physical function and disability within a median time of 137 days. A minority $(12 \%)$ needs further follow up and rehabilitation maintenance due to incomplete recovery.

\section{References}

1. Lian J, Jin X, Hao S, Jia H, et al. Epidemiological, clinical, and virological characteristics of 465 hospitalized cases of coronavirus disease 2019 (COVID-19) from Zhejiang province in China. Influenza Other Respir Viruses 2020;14:564-574.

2. Lian J, Jin X, Hao S, Cai H, et al. Analysis of epidemiologic and clinic features in older patients with coronavirus disease 2019 (COVID-19) outside Wuhan. Clin Infect Dis 2020;71:740-7.

3. Beeching NJ, Fletcher TE, Fowler R. Coronavirus disease 2019 (COVID-19). BMJ Best Practices 2020. Accessed on: 31 December 2020. Available from: https://bestpractice.bmj. com/topics/en-gb/3000168

4. Belli S, Balbi B, Prince I, et al. Low physical functioning and impaired performance of activities of daily life in COVID-19 patients who survived hospitalisation. Eur Respir J 2020;56:2002096.

5. Paneroni M, Simonelli C, Saleri M, et al. Muscle strength and physical performance in patients without previous disabilities recovering from COVID-19 pneumonia. Am J Phys Med Rehabil 2021;100:105-9.

6. Huang C, Huang L, Wang Y, et al. 6-month consequences of COVID-19 in patients discharged from hospital: a cohort study. Lancet 2021;397:220-32.

7. Liu K, Zhang W, Yang Y, et al. Respiratory rehabilitation in elderly patients with COVID-19: A randomized controlled study. Complement Ther Clin Pract 2020;39:101166.

8. Lazzeri M, Lanza A, Bellini R, et al. Respiratory physiotherapy in patients with COVID-19 infection in acute setting: a Position Paper of the Italian Association of Respiratory Physiotherapists (ARIR). Monaldi Arch Chest Dis 2020;90:1285.

9. Vitacca M, Carone M, Clini EM, et al. Joint statement on the role of respiratory rehabilitation in the COVID-19 crisis: The Italian position paper. Respiration 2020;99:493-9.

10. Maltais F, LeBlanc P, Jobin J, et al. Intensity of training and physiologic adaptation in patients with chronic obstructive pulmonary disease. Am J Respir Crit Care Med 1997;155:555-61.

11. Guralnik JM, Simonsick EM, Ferrucci L, et al. A short physical performance battery assessing lower extremity function: association with self-reported disability and prediction of mortality and nursing home admission. J Gerontol 1994;49:M85-94.

12. Shah S, Vanclay F, Cooper B. Improving the sensitivity of the Barthel Index for stroke rehabilitation. J Clin Epidemiol 1989;42:703-9.

13. Gandotra S, Lovato J, Case D, et al. physical function trajectories in survivors of acute respiratory failure. Ann Am Thorac Soc 2019; 6:471-7. 